\title{
Estrategias para la Conservación y Uso Sustentable de la Diversidad Biológica
}

\author{
Autor: Nixon Alexander Maldonado Valero \\ Universidad Pedagógica Experimental Libertador, UPEL \\ nixonmaldonadova@gmail.com \\ Barinas, Venezuela \\ https://orcid.org/0000-0003-1123-5553
}

\section{Resumen}

Está investigación tuvo como propósito desarrollar estrategias para la conservación y uso sustentable de la diversidad biológica en la Escuela Técnica Agropecuaria Nacional (ETAN) "Don Simón Rodríguez", del Municipio Pedraza del Estado Barinas. Se caracteriza por ser un estudio cualitativo en la modalidad de investigación acción y sustentado en un diseño de campo. El escenario fue la Escuela Técnica Agropecuaria Nacional "Don Simón Rodríguez", los informantes claves están constituidos por (2) docentes y (1) obrero de la institución. La técnica utilizada fue la entrevista en profundidad, el instrumento utilizado fue un guión para establecer las unidades temáticas. Con respecto al tratamiento de los resultados se categorizó, contrastó y teorizó la información obtenida de los relatos de las personas inmersas en el proceso investigativo, se elaboró un diagnóstico sobre el manejo de las áreas boscosas de la institución. Posteriormente se elaboró la propuesta denominada Plan de acción para la conservación y uso sustentable de la diversidad biológica. Asimismo, se desarrolló el plan preconcebido en donde los participantes consideraron que las acciones ejecutadas permitieron adquirir conocimientos primordiales sobre la biodiversidad y el uso sustentable de los recursos naturales.

Palabras clave: conservación ambiental; desarrollo sostenible; diversidad biológica. 


\title{
Strategies for the Conservation and Sustainable Use of Biological Diversity
}

\begin{abstract}
Research is intended to develop strategies for the conservation and sustainable use of biodiversity in the National Agricultural School of Technology (ETAN) "Don Simon Rodriguez", of the Municipality Pedraza del Estado Barinas. It is characterized by being a qualitative study in the field of action research and supported in a field design. The stage was the National Agricultural School of Technology "Don Simon Rodriguez", the key informants are composed of (2) teachers and (1) a worker of the institution. The technique used was the interview in depth, the instrument used was a guide to establishing the thematic units. Regarding the treatment of the results, the information obtained from the reports of the immersed persons in the investigative process was categorized, contrasted and theorized, a diagnosis was carried out on the management of the woodland areas of the institution. Subsequently, the proposal called Action plan for the conservation and sustainable use of biological diversity was developed. Similarly, the preconceived plan was developed where participants considered that the executed actions allowed the acquisition of primary knowledge of biodiversity and the sustainable use of natural resources.
\end{abstract}

Keywords: environmental conservation; sustainable development; biological diversity. 


\section{Introducción}

Durante siglos la humanidad se ha apoderado intensa e irracionalmente de los recursos naturales hasta el punto de arriesgar la vida misma del planeta, poniendo en peligro la diversidad biológica. La ambición y las ansias de riqueza han provocado cambios irreversibles a los ecosistemas naturales amparados en la bandera de la industrialización. De modo que, Frers (2008), afirma al respecto:

La extinción de especies animales y vegetales constituye un proceso irreversible que nos priva para siempre de un material genético, único e irremplazable, del que tal vez ni siquiera sepamos aún, qué aplicaciones prácticas podrá tener en beneficio de la misma humanidad que los destruye (pág. 2).

Resultan evidentes y palpables los impactos sobre la calidad de vida a causa del deterioro ambiental, pero lo más grave aún, es que nos detengamos a plantear un cambio de conducta y pensamiento, cuando quizás se agoten los recursos naturales que nos dan la vida y por tanto, corregir el modelo irracional de consumo, sea tarde e insignificante. Es de resaltar que García, López y Ramírez (2015), señalan que: "los sistemas naturales y la diversidad biológica, proveen a la sociedad de una gran variedad de bienes y servicios ambientales, regulación hídrica, regulación del clima, polinizadores, dispersores, para el desarrollo y bienestar de las poblaciones humanas" (pág. 2); lo que es esencial para el establecimiento de una relación armónica entre el hombre y el ambiente.

En función de matizar parte de esta situación, Betancourt y Moreno (2015a), plantean que: "dentro de los ecosistemas boscosos, uno de los sistemas más amenazados a nivel mundial son los bosques tropicales, caracterizados por albergar el $65 \%$ de la riqueza mundial de especies animales y vegetales" (pág. 273). De acuerdo con esta opinión es fundamental desarrollar acciones para la promoción de estrategias y categorías de manejo 
apropiadas para conservar la biodiversidad, como la creación de bosques protegidos.

Es en definitiva fundamental, considerar que un ambiente sano solo puede ser posible, mediante la colaboración particular de cada habitante de la tierra, para lo cual profundizar en un proceso de sensibilización desde casa y desde las instituciones educativas, industrias y empresas dependientes de materias primas provenientes de los recursos naturales; es indispensable. Para ello se deben practicar claves sencillas y realmente hábiles en el ahorro de la energía eléctrica, el agua potable; dar buen uso y provecho a los desechos domésticos y disminuir prácticas como la caza de animales exóticos, deforestación y quema irracional.

Es incuestionable que los cambios suscitados en los últimos tiempos a escala mundial, en esferas económica, social y política, vienen generando ritmos de vida y condiciones ambientales que han logrado resolver algunas complicaciones; pero no es menos cierto que han afectado la calidad ambiental, revirtiéndose en la atroz y acelerada pérdida de condiciones vitales para la existencia humana y la diversidad biológica, contaminación y destrucción del planeta; quien hoy, se sacude y reclama por su derecho universal a la vida. Con respecto al tema, Betancourt y Moreno (2015b), expresan:

Los bosques en Venezuela ocupaban en 2010 un 50,49\% (46.275.000 ha.), de la superficie del territorio (91.644.500 ha), lo que colocaba a este país entre los 45 países del mundo donde más del $50 \%$ de su territorio estaba ocupado por superficie forestal. En Venezuela son pocas las áreas donde se protegen o manejan los ecosistemas boscosos, especialmente los presentes en la ecorregión de los Llanos (pág. 277).

Por su puesto que todos los datos anteriores producen inmensa congoja, al observar actualmente extensos pastizales donde otrora existían zonas boscosas repletas de una muy única y rica diversidad biológica; 
escenario realmente alarmante que debe motivar a la ciudadanía, al gobierno $y$ al Estado a ejercer un substancial cambio en procura de conservar un ambiente sano y tomar medidas efectivas y urgentes para en general, poner freno a toda situación adversa a la existencia armónica entre la raza humana y la naturaleza.

De esta realidad no escapa el estado Barinas, entidad federal perteneciente a la ecorregión de los llanos venezolanos, donde existían dos reservas forestales importantes, Caparo y Ticoporo que fueron explotadas y vejadas por empresas madereras hasta el extremo de solo observar hoy en día, lagunas piscícolas, pastizales y el monocultivo de teca en pocas fincas ganaderas. Además de ello, se deforestan y fragmentan bosques naturales privatizados por productores agropecuarios quienes suman sus hectáreas a tierras de cultivo y ganadería, evidenciándose muy pocas áreas naturales realmente protegidas.

Hay que tener en cuenta que en el estado Barinas existen nueve Escuelas Técnicas Agropecuarias poseedoras de bondadosas tierras fértiles y excepcionales recursos naturales cuya razón de ser como instituciones educativas en la modalidad Media Técnica es la educación en los aspectos técnicos para la producción y transformación de las materias primas provenientes de actividades del campo.

De modo que el documento Escuelas Técnicas Robinsonianas del Ministerio de Educación y Deportes (2004): plantea que sus egresados serán "activistas celosos e implacables de la defensa del ambiente, comprometidos con el desarrollo humano y el bienestar de la población" (pág. 68). Empero, la correspondencia entre lo evidenciado y el deber ser conservacionista de la educación formal se pone en riesgo en Escuelas Técnicas, cuyas prácticas no se corresponden con la formación de ese activista.

Sin embargo, y en contraposición a lo señalado en párrafos anteriores, se destaca que prácticas como la deforestación y fragmentación de 
ecosistemas, no son ajenas a la Escuela Técnica Agropecuaria Nacional (ETAN) "Don Simón Rodríguez", institución educativa ubicada en la población de Palma Sola II, a ocho kilómetros $(8 \mathrm{~km})$ del balneario "La Acequia", Parroquia Ciudad Bolivia, Municipio Pedraza del Estado Barinas, donde el autor del presente estudio, ha podido observar prácticas que indican sin equívocos, ser causantes de problemas ecológicos de fatales consecuencias.

Por ejemplo, en el año 2013 se introdujo en la Escuela Técnica Agropecuaria Nacional "Don Simón Rodríguez", maquinaria pesada deforestando y convirtiendo en área agrícola un aproximado de cinco hectáreas ( 5 ha) de bosque natural, procediendo a quemar una vez seco en trincheras; contribuyendo de esta manera al recalentamiento del planeta, a la merma de especies que perdieron su hábitat natural y en definitiva fragmentando y rompiendo un ecosistema que coexistía armoniosamente.

En tal sentido, se deduce la urgente y extraordinaria necesidad de sensibilizar tanto a las autoridades como a los actores intervinientes en el hecho educativo de la Escuela Técnica Agropecuaria Nacional (ETAN) "Don Simón Rodríguez", (sus docentes, estudiantes, obreros, vecinos, representantes y a la comunidad donde está inserta), en favor de implementar la estrategia y la categoría de manejo más certera, que oriente a la protección de la diversidad de vida existente en la institución.

\subsection{Propósito de la investigación}

Desarrollar estrategias para la conservación y uso sustentable de la diversidad biológica en la Escuela Técnica Agropecuaria Nacional (ETAN) "Don Simón Rodríguez", del Municipio Pedraza del Estado Barinas.

\subsection{Teoría y conceptos}

La teoría constructivista se define como la forma mediante la cual el hombre construye su propio conocimiento partiendo de sus experiencias, 
según Ortiz (2015):

El conocimiento es una construcción del ser humano: cada persona percibe la realidad, la organiza y le da sentido en forma de constructos, gracias a la actividad de su sistema nervioso central, lo que contribuye a la edificación de un todo coherente que da sentido y unicidad a la realidad (pág. 5).

Todas las personas tienen la capacidad de construir su propio conocimiento partiendo de las estructuras mentales ya establecidas, de allí que el constructivismo aporta estrategias novedosas y útiles a los procesos pedagógicos, la educación plantea el escenario para que el aprendiz desarrolle sus propias estructuras cognoscitivas.

\subsection{Diversidad biológica}

Para esta investigación la definición de biodiversidad es fundamental para comprensión del estudio en escena y es por lo que se adopta la conceptualización realizada por Dorado (2010), el cual señala:

La biodiversidad abarca, por tanto, la enorme variedad de formas mediante las que se organiza la vida. Incluye todas y cada una de las especies que cohabitan con nosotros en el planeta, sean animales, plantas, virus o bacterias, los espacios o ecosistemas de los que forman parte y los genes que hacen a cada especie, y dentro de ellas a cada individuo, diferente del resto (pág. 8).

De acuerdo con esta definición es esencial el desarrollo de nuevas técnicas de conservación para lograr que se preserve la vida en el transcurrir de los tiempos y garantizando el resguardo de su potencial genético.

\subsection{Recursos naturales}

El hombre siempre ha requerido de la naturaleza para cubrir sus prioridades, modificando el ambiente y adecuándolo a su conveniencia, por eso, es importante desarrollar actividades orientadas a su conservación, según 
Blanco y Rey (2016): los recursos naturales son "cualquier forma de materia o energía que existe de modo natural y que puede ser utilizada por el ser humano" (pág. 6); de manera que es importante hacer uso racional de ellos para garantizar su preservación en el tiempo.

\subsection{Deforestación}

Contribuye a la destrucción de muchos ecosistemas, repercutiendo en la pérdida de diversidad biológica, cada día son menos los bosques que cubren la faz de la tierra, y el calentamiento global y las consecuencias que este produce cada vez son más sentidas. El Programa de las Naciones Unidas para el Medio Ambiente (2015), indica:

La deforestación es desmontar total o parcialmente las formaciones arbóreas para dedicar el espacio resultante a fines agrícolas, ganadero o de otro tipo. Esta concepción no tiene en cuenta ni la pérdida de superficie arbolada por desmonte parcial, ni el entresacado selectivo de maderas, ni cualquier otra forma de degradación (pág. 3).

De acuerdo con lo expuesto anteriormente la pérdida de zonas boscosas es preocupante, por la pérdida considerable de diversidad biológica porque presenta consecuencias graves para los ecosistemas, además que se disminuye la capacidad productiva de los suelos, así como también se ve limitada la capacidad de purificación del aire.

\subsection{Conservación ambiental}

La conservación en un sentido de empatía por cuidar lo propio, lo autóctono con el fin de hacer uso de los recursos evitando su agotamiento, según Bonilla (2016): "la conservación ambiental es el uso racional y sostenible de los recursos naturales y el ambiente, teniendo como objetivos garantizar la perseverancia de las especies en sus ecosistemas, esto permitirá mejorar la calidad de vida de las poblaciones" (pág. 44). La conservación tiene 
una importancia de primer orden para la humanidad.

\section{Metodología}

\subsection{Naturaleza del estudio}

Esta investigación se encuadra en los parámetros de la metodología cualitativa, según Grijalva y Mancheno (2012): "una aproximación sistémica que permite describir las experiencias de la vida y darles significado. Su objetivo es ver los acontecimientos, acciones, normas, valores, etc. desde la perspectiva de la persona que está siendo estudiada" (pág. 1). Se selecciona esta metodología porque permite comprender en profundidad el tema que se investiga.

\subsection{Tipo de investigación}

El presente trabajo investigativo encaja en forma adecuada en la modalidad de investigación - acción, la cual permite ir desarrollando nuevas estrategias a medida que el proceso indagatorio avance. En este sentido, Sequera (2014), expone:

La Investigación Acción es considerada en la actualidad social del conocimiento como una metodología que forja una elevada capacidad para dar respuestas a los diferentes problemas que se presentan en la cotidianidad, en tanto que la misma tiene su accionar en el contexto de la comunidad y no solo involucra al investigador, sino también a todos los integrantes del mismo, los cuales a partir de la detección de la situación problemática, aportarán sus ideas y posibles soluciones desde la elaboración de proyectos o planes de acción (pág. 224).

Lo importante de esta modalidad es la flexibilidad que ofrece al investigador de hacer ajustes sobre la marcha, corrigiendo y afinando los detalles necesarios para garantizar el alcance de los propósitos establecidos. 


\subsection{Diseño de la investigación}

De acuerdo con las particularidades del estudio se seleccionó la investigación de campo como diseño, por ser la que va directamente al escenario donde ocurren los hechos, se busca obtener información veraz y fiable desde las perspectivas de las personas que comparten un espacio geográfico y una misma cultura, es importante que la ruta metódica para la obtención de evidencias consistentes esté bien diseñada para alcanzar el propósito del estudio. Con relación al tema, Barrios (2014), expresa:

Se entiende por investigación de campo, el análisis sistemático de problemas en la realidad, con el propósito bien sea de describirlos, interpretarlos, entender su naturaleza y factores constituyentes, explicar sus causas y efectos, o predecir su ocurrencia, haciendo uso de métodos característicos de cualquiera de los paradigmas o enfoques de investigación conocidos o en desarrollo. Los datos de Interés son recogidos en forma directa de la realidad; en este sentido se trata de investigaciones a partir de datos originales o primarios (pág. 18).

En relación con lo expuesto anteriormente, este diseño es fundamental para los propósitos de la investigación realizada, constituyó la vía expedita para la recolección de las expectativas de las personas de la comunidad referente a la conservación de la biodiversidad. Lo fundamental del trabajo de campo es que permite revisar los resultados y en caso de ser necesario volver a las fuentes primarias para verificar la autenticidad de los testimonios.

\subsection{Escenario e informantes claves}

En esta etapa se describe de manera específica el espacio geográfico y las características de las personas seleccionadas como informantes clave, como escenario se seleccionó la Escuela Técnica Agropecuaria Nacional (ETAN) "Don Simón Rodríguez", del Municipio Pedraza del Estado Barinas.

Para este proceso de investigación se escogieron tres (3) informantes, dos (2) 
docentes y un (1) obrero de la institución cuyos criterios coinciden en que son habitantes de la Comunidad de Palma Sola desde hace más de cuarenta y cinco años, son trabajadores activos de la ETAN "Don Simón Rodríguez", conocen suficientemente la existencia de los bosques de la zona y poseen actitudes reconocidas de trabajar por convicción de vida en defensa de los derechos y deberes ambientales en el ámbito escolar y comunitario.

\subsection{Técnicas e instrumentos para la recolección de información}

Para este estudio se seleccionó la entrevista en profundidad, siendo esta técnica las más utilizadas en estudios cualitativos, en especial en investigaciones donde se busca diagnosticar una situación particular de naturaleza social y educativa, a lo cual, Salinas (2013), plantea que: “...como técnica de carácter cualitativa, dialógica, posibilita acceder en forma privilegiada a los discursos o a los procesos comunicacionales y la construcción de la cultura" (pág. 1). La entrevista estuvo alineada por un guión de preguntas semiestructuradas para plantear las unidades temáticas.

\subsection{Validez y fiabilidad del instrumento}

La validez de este estudio reposa en el reflejo claro de la situación que se observa. Según Martínez (2006): "La validez de instrumentos cualitativos es un proceso reflexivo y metódico que responde al establecimiento de la categorización, contrastación, estructuración y teorización” (pág. 3). Es por ello que los instrumentos utilizados recogieron la información que realmente se requería. El presente estudio demostró la fiabilidad en la concordancia de sus resultados, a través de la triangulación. Según Ávila (2010): "La triangulación es una técnica en donde se usan 30 más perspectivas o diferentes observadores, o varias fuentes de datos, los cuales pueden ser cualitativos/cuantitativos distintos" (pág. 1). 


\subsection{Técnicas de análisis de información}

Las técnicas para analizar los datos cualitativos de esta investigación comprendieron el siguiente proceso:

Categorización: Martínez (2008a), afirma sobre este término: "Es decir clasificar, conceptualizar o codificar mediante un término o expresión que sea claro e inequívoco (categoría descriptiva), el contenido o idea central de cada unidad temática" (pág. 77). El proceso de categorización se realizó de la siguiente forma: en primera instancia se releyeron las entrevistas subrayando las palabras más relevantes. Luego se dividió el contenido en unidades temáticas.

Contrastación: Según Urbano, Requena y Rivas (2015): este proceso consiste en "relacionar y contrastar los resultados con aquellos estudios similares que se presentaron el marco teórico referencial, para ver cómo aparecen de las perspectivas diferentes o sobre marcos teóricos más amplios y explicar mejor lo que el estudio verdaderamente significa" (pág. 3). En este estudio se contrastaron los testimonios de los informantes claves con el marco teórico establecido previamente.

Teorización: Martínez (2008b): también plantea que una teoría es "una construcción mental simbólica verbal o icónica de naturaleza conjetural o hipotética, que nos obliga, de nuevo a completar, integrar, unificar, verificar, sistematizar un cuerpo de conocimientos que hasta el momento se consideraban incompletos, imprecisos, inconexos o intuitivos" (pág. 99). En esta investigación la teoría surgió del contraste entre la información recopilada de los informantes claves y el marco teórico establecido.

\section{Resultados}

Según los relatos de los informantes se evidencia que poseen un amplio conocimiento acerca de la fauna de la localidad e identifican una importante lista de animales silvestres y autóctonos de la zona, que corren el peligro de 
perder su habitad sino se cuidan las áreas boscosas.

De igual forma, se evidenció la existencia de flora autóctona de la región, enfatizándose sobre la conservación de especies vegetales como el mijao "Anacardium excelsum" y el balso "Ochroma pyramidale" los cuales son especies que albergan a la gran mayoría de la fauna silvestre de la zona, además de ser reguladores micro climáticos.

Los relatos de los entrevistados demuestran que la deforestación disminuye la diversidad biológica de las zonas intervenidas. En el caso de la fauna provoca desequilibrios y el éxodo de especies nativas a otras zonas para garantizar la sobrevivencia.

La sustentabilidad es necesaria para la preservación del planeta, sin embargo, en la investigación realizada, los entrevistados reconocieron el mal manejo que se ha hecho con las zonas boscosas de la institución en estudio exponiendo que ha provocado cambios en el microclima, también opinan que es necesario tomar medidas para que estas acciones perjudiciales para el ambiente no vuelvan ocurrir.

De acuerdo con los relatos de los informantes clave, se reiteró la premisa que para promover valores ambientales, es necesaria la práctica, se requiere de una nueva visión ecologista y en especial en la Escuela Técnica Agropecuaria Nacional (ETAN) "Don Simón Rodríguez", se deben desarrollar tecnologías amigables con el ambiente. Este tipo de instituciones deben fomentar el manejo sustentable de la diversidad biológica.

Con respecto a la formación ambiental los entrevistados estuvieron de acuerdo con realizar un plan de acción para el diseño y aplicación de estrategias para para la conservación y uso sustentable de la diversidad biológica, lo cual es importante porque contribuye con el propósito de la investigación que busca la participación activa de las personas inmersas en la problemática en estudio. 


\section{Conclusiones}

En la Escuela Técnica Agropecuaria Nacional "Don Simón Rodríguez", se evidenció que las personas que allí laboran, poseen un amplio conocimiento acerca de la fauna de la localidad, lo cual es importante porque se demuestra la presencia de animales silvestres autóctonos de la zona que corren el peligro de perder su habitad sino se cuidan las áreas boscosas. Asimismo, se realizó énfasis en la preocupación por el cuidado de especies vegetales como el mijao y el balso.

El estudio demostró que la deforestación disminuye la diversidad biológica de las zonas intervenidas. En el caso de la fauna provoca desequilibrios y el éxodo de especies nativas a otras zonas para garantizar la sobrevivencia. Se reiteró la siguiente premisa, para promover valores ambientales, es necesaria la práctica, se requiere de una nueva visión ecologista y en especial en la institución, se deben desarrollar tecnologías amigables con el ambiente. Este tipo de instituciones debe fomentar el manejo sustentable de la diversidad biológica. Con respecto a la formación ambiental los entrevistados estuvieron de acuerdo con realizar un plan de acción para el diseño y aplicación de estrategias para para la conservación y uso sustentable de la diversidad biológica.

Para el desarrollo de estrategias para la preservación de la biodiversidad se consultaron fuentes documentales y electrónicas, se abordaron algunas personas de la comunidad con habilidades ecológicas. Después de organizar los contenidos los materiales y el talento humano se diseñó el Plan acción denominado estrategias para la conservación y uso sustentable de la diversidad biológica en la Escuela Técnica Agropecuaria Nacional (ETAN) "Don Simón Rodríguez", el cual se fundamentó en la formación de las personas con relación al aprovechamiento sustentable de las áreas boscosas de la institución.

Con respecto a la aplicación del plan antes mencionado, fue 
promocionado por los educadores de la institución, se ejecutaron los talleres con la participación de docentes, estudiantes, obreros y personas de la comunidad. La experiencia fue muy productiva, los participantes demostraron interés por el logro de los propósitos planteados y se desarrollaron propuestas interesantes para continuar con el trabajo ecologista.

Con respecto a la evaluación del plan, fue catalogado como bueno e importante, a través de los talleres se realizó el intercambio de experiencias y reflexiones para el uso responsable de las áreas boscosas de la Escuela Técnica Agropecuaria Nacional "Don Simón Rodríguez", de allí surgió la propuesta de preservar el bosque de la institución como área de protección bajo regímenes especiales.

\section{Referencias}

Ávila, B. (2010). Triangulación. La triangulación, una técnica de investigación. Colombia: Informativo CEAD Barranquilla. [Documento en línea]. Recuperado de:

http://triangulacion-tecnicadeinvest.blogspot.com/

Barrios, M. (2014). Manual de trabajos de grado de especialización y maestría y tesis doctorales. Caracas, Venezuela: Fondo Editorial de la Universidad Pedagógica Experimental Libertador - FEDUPEL.

Betancourt, A., \& Moreno, E. (2015a,b). Corredores ecológicos como estrategia para la conservación de los ecosistemas boscosos de la reserva forestal de Caparo. Barinas, Venezuela: Universidad Nacional Experimental de los Llanos "Ezequiel Zamora" - UNELLEZ.

Blanco, A., \& Rey, M. (2016). Influencia de la distribución de los recursos naturales en el desarrollo regional de Venezuela. Caracas, Venezuela: Universidad Central de Venezuela.

Bonilla, D. (2016). El reciclaje como estrategia didáctica para la conservación ambiental (Proyecto en ejecución). Revista Scientific, 
1(1), 36-52. Recuperado de:

https://doi.org/10.29394/scientific.issn.2542-2987.2016.1.1.3.36-52

Dorado, A. (2010). ¿Qué es la biodiversidad? Madrid, España: Greenpeace.

Frers, C. (2008). Cuidemos la Biodiversidad. Argentina: Ecoportal. [Documento en línea]. Recuperado de:

https://www.ecoportal.net/paises/bano-portatil-que-almacena-residuosy-produce-energia/

García, M., López, J., \& Ramírez, M. (2015). Regeneración natural de la vegetación como base para el desarrollo de estrategias de restauración ecológica en tres Biotopos protegidos en la Reserva de Biosfera Maya, Guatemala. Ciencia, Tecnología y Salud, 2(1), 5364, ISSN: 2410-6356. Recuperado de:

http://digi.usac.edu.gt/ojsrevistas/index.php/cytes/article/download/48/6 $\underline{2}$

Grijalva, O., \& Mancheno, S. (2012). Investigación cualitativa. Quito, Ecuador: Universidad Central del Ecuador. Recuperado de:

https://www.monografias.com/trabajos95/investigacioncualitativa/invest igacioncualitativa.shtml

Martínez, M. (2008a,b). La investigación cualitativa etnográfica en educación. México: Editorial Trillas.

Martínez, M. (2006). Validez y confiabilidad en la metodología cualitativa. Revista Paradigma, 27(2), 1-20, ISSN: 1011-2251. Recuperado de: http://revistas.upel.edu.ve/index.php/paradigma/article/view/3759/1866 Ministerio de Educación y Deportes (2004). Escuelas Técnicas Robinsonianas. Caracas, Venezuela: Talleres de Intenso.

Ortiz, D. (2015). El constructivismo como teoría y método de enseñanza. Sophia, Colección de Filosofía de la Educación, (19), 93-110, ISSN: 1390-3861. Recuperado de:

http://www.redalyc.org/articulo.oa?id=441846096005 
Programa de las Naciones Unidas para el Medio Ambiente (2015). Informe Anual de 2015. Núm. de trabajo: DCP/1938/NA, ISBN: 978-92-8073518-5. Estados Unidos: PNUMA. Recuperado de: https://wedocs.unep.org/bitstream/handle/20.500.11822/7544/UNEP A nnual Report 20152016UNEPAR2015 ES web.pdf.pdf?sequence $=1$ 1 \&isAllowed $=y$

Urbano, A., Requena, C., \& Rivas, M. (2015). Estructura de la investigación. República Bolivariana de Venezuela: Universidad Pedagógica Experimental Libertador, Instituto de Mejoramiento Profesional del Magisterio, Extensión Tucupita. [Documento en línea]. Recuperado de:

https://es.slideshare.net/Maricarmenrivas1976/categorizacin-45777380 Salinas, P. (2013). La entrevista en profundidad: una estrategia de comprensión del discurso minero en el norte de Chile. Actas del $2^{\circ}$ Congreso Nacional sobre Metodología de la Investigación en Comunicación, ISBN: 978-84-616-4124-6. España: Universidad de Valladolid, Facultad de Ciencias Sociales, Jurídicas y de la Comunicación, págs. 543-562. Recuperado de:

https://dialnet.unirioja.es/descarga/articulo/4229106.pdf

Sequera, M. (2014). Investigación acción: un método de investigación educativa para la sociedad actual. ARJÉ. Revista de postgrado FaCE-UC, 10(18), 223-229, ISSN-e: 2443-4442; ISSN: 1856-9153. Recuperado de: http://arje.bc.uc.edu.ve/arj18/art23.pdf 


\section{Nixon Alexander Maldonado Valero}

e-mail: nixonmaldonadova@gmail.com

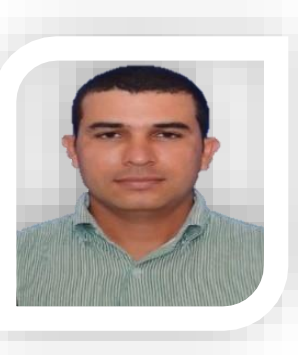

Nacido en ciudad Bolivia, estado Barinas, Venezuela.

Profesor en Educación Agropecuaria egresado de la

Universidad Pedagógica Experimental Libertador.

Miembro de la Asociación Protectora a la Vida

(Asoprovida). Docente en Función Supervisora en la

Red Intercircuital de Educación en el municipio Pedraza, con antigüedad de doce años en servicio.

El contenido de este manuscrito se difunde bajo una Licencia de Creative Commons ReconocimientoNoComercial-Compartirlgual 4.0 Internacional 